\title{
Fourier-Transform Infrared Spectroscopy of Epiretinal Membranes and Internal Limiting Membranes after Pars Plana Vitrectomy with Membrane Peeling
}

\author{
Christoph Leisser ${ }^{\mathrm{a}}$ Eleftherios Paschalis ${ }^{\mathrm{b}}$ Stamatia Rokidi $^{\mathrm{b}}$ \\ Martina Behanova $^{b}$ Manuel Ruiss $^{a}$ Wilhelm Burgmüller ${ }^{a}$ Oliver Findl ${ }^{a}$

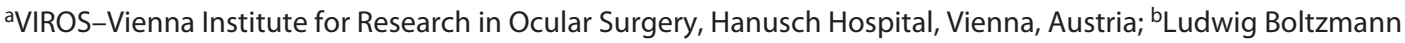 \\ Institute of Osteology at 1st Medical Department, Hanusch Hospital of Österreichische Gesundheitskasse (ÖGK) and \\ Research funds of the Austrian workers compensation board (AUVA) Trauma Centre Meidling, Vienna, Austria
}

\section{Keywords}

Fourier-transform infrared spectroscopy · Epiretinal membrane $\cdot$ Internal limiting membrane $\cdot$ Vitrectomy with membrane peeling

\begin{abstract}
Introduction: Fourier-transform infrared imaging (FTIRI) enables examination of protein secondary structure in the analyzed tissues. The aim of our study was to examine the distribution of secondary structures in epiretinal membranes (ERMs) and internal limiting membranes (ILMs), and to explore possible associations to other diagnostic variables. Methods: This prospective pilot study included patients scheduled for pars plana vitrectomy with membrane peeling. ERMs and ILMs were harvested during surgery and placed on a $\mathrm{BaF}_{2}$ window for postsurgical FTIRI analysis. Infrared hyperspectral images were subjected to second and fourth derivative analysis to obtain information of the protein secondary structures present in the tissues. Results: Samples of 43 patients were analyzed, with the triple helical domain showing the highest prevalence in the examined tissues. The other secondary structures (beta-sheet, random coil, and beta-turn) showed a heterogenous distribution in
\end{abstract}

karger@karger.com www.karger.com/ore

Karger!
(C) 2021 The Author(s)

Published by S. Karger AG, Basel

This is an Open Access article licensed under the Creative Commons Attribution-NonCommercial-4.0 International License (CC BY-NC) (http://www.karger.com/Services/OpenAccessLicense), applicable to the online version of the article only. Usage and distribution for commercial purposes requires written permission. the examined samples, without specific associations to indication of surgery, comorbidities, outcomes from optical coherence tomography, and intrasurgical findings. Conclusions: $\mathrm{FTIRI}$ enables analysis of the spatial distribution of protein secondary structures in the examined tissues; thus, it is a useful analytical technique for the analysis of ERMs and ILMs.

(C) 2021 The Author(s)

Published by S. Karger AG, Basel

\section{Introduction}

Fourier-transform infrared spectroscopy imaging (FTIRI) is a vibrational spectroscopic method suitable for the analysis of proteins that show vibrational band characteristics of peptide groups and side chains of proteins. The amide I band is especially sensitive to secondary structures [1], and by Fourier transformation, raw infrared spectra can be assigned to specific conformations, such as beta-sheet domain, random coil domain, alphahelical domain, triple helical domain, and beta-turn domain [2].

Epiretinal membranes (ERMs), a pathological condition leading to metamorphopsia and disturbance in vi- 
Table 1. Distribution of secondary structures of proteins in ERM and ILM

\begin{tabular}{llllll}
\hline$(n)$ & $\begin{array}{l}\text { Beta-sheet } \\
\text { domain, \% }(n)\end{array}$ & $\begin{array}{l}\text { Random coil } \\
\text { domain, \% }(n)\end{array}$ & $\begin{array}{l}\text { Alpha-helical } \\
\text { domain, \% }(n)\end{array}$ & $\begin{array}{l}\text { Triple helical } \\
\text { domain, \% }(n)\end{array}$ & $\begin{array}{l}\text { Beta-turn } \\
\text { domain, \% }(n)\end{array}$ \\
\hline iERM (17) & $47(8)$ & $18(3)$ & $24(4)$ & $88(15)$ & $71(12)$ \\
dERM (9) & $67(6)$ & $11(1)$ & $22(2)$ & $100(9)$ & $78(7)$ \\
FTMH (7) & $29(2)$ & $14(1)$ & $14(1)$ & $86(6)$ & $43(3)$ \\
Other ILM samples (4) & $50(2)$ & $0(0)$ & $0(0)$ & $100(4)$ & $50(2)$ \\
Atrophic LMH (3) & $67(2)$ & $33(1)$ & $33(1)$ & $100(3)$ & $100(3)$ \\
ERM foveoschisis (4) & $50(2)$ & $0(0)$ & $25(1)$ & $75(3)$ & $50(2)$ \\
Pseudomacular hole (2) & $0(0)$ & $0(0)$ & $0(0)$ & $100(2)$ & $0(0)$ \\
\hline
\end{tabular}

ERM, epiretinal membrane; iERM, idiopathic epiretinal membrane; dERM, diabetic epiretinal membrane; FTMH, full thickness macular hole; LMH, lamellar macular hole; ILM, internal limiting membrane.

sual acuity, and the internal limiting membrane (ILM), the basement membrane of Muller cells at the vitreous side, could be shown by immunohistochemical analysis to be rich in different types of collagen and other proteins [3-6]. These membranes, therefore, are a potential substrate for FTIRI; nevertheless, to our knowledge, no studies of FTIRI analysis of ERMs and ILM exist to date. The aim of our study was to use FTIRI to examine the distribution of specific secondary structures, in ERMs and ILM, and to explore possible associations with other diagnostic outcomes.

\section{Materials and Methods}

This prospective study included patients scheduled for pars plana vitrectomy with membrane peeling between August 2017 and December 2018 at the ophthalmic department of the Hanusch Hospital in Vienna, Austria. The inclusion criteria were age above 18 years, presence of an ERM with indication for surgery (defined as significant loss of vision and/or metamorphopsia due to the ERM), full thickness macular hole, and lamellar macular hole. All research and measurements followed the tenets of the Declaration of Helsinki, and the study was approved by the local Ethics Committee of the city of Vienna (Clinical trials registration: NCT03452748). Written informed consent was obtained from all patients in the study.

Surgery was performed in a standardized fashion with $23 \mathrm{G}$ pars plana vitrectomy and membrane peeling by an experienced vitreoretinal surgeon. To visualize the ERM and ILM, chromovitrectomy with trypan blue and brilliant blue G-based dyes (MembraneBlue-Dual; D.O.R.C. (Dutch Ophthalmic Research Center), Rotterdam, The Netherlands) was performed. The ERM and ILM were peeled using an end-gripping forceps, with the starting point typically temporal to the macula. Excised ERM and when possible ILM were harvested and placed on $\mathrm{a} \mathrm{BaF}_{2}$ window for postoperative FTIRI. All patients received nonsteroidal anti-inflammatory eye drops and steroidal eye drops during the first month after surgery.
Phacovitrectomy with implantation of an intraocular lens was performed only in case of coexisting vision-affecting cataract.

FTIRI analysis was performed by using a Bruker Tensor 27 spectrometer (Bruker Optics, Ettlingen, Germany) attached to an infrared microscope (Hyperion 3000; Bruker Optics), equipped with an infrared array detector (Mercury Cadmium Telluride; $64 \times 64$ array) affording a lateral spatial resolution of about $3.9 \times$ $3.9 \mu \mathrm{m}^{2}$. Spectra were acquired with a resolution of $8 \mathrm{~cm}^{-1}$. Tissue samples were placed on $\mathrm{BaF}_{2}$ windows and examined in their native state (no tissue processing involved). The raw infrared spectra were further processed by second derivative analysis to elucidate the number and exact position of the underlying peaks that can be assigned to specific secondary structures. In instances where it was obvious that the calculated second derivative peaks were not single but still composite ones (presence of discernible shoulders), 4th derivative spectra were calculated as well.

Continuous data are described using mean and standard deviation, and for categorical data, absolute frequencies and percentages are presented. To examine associations of FTIRI outcomes to diagnosis and general factors, $\chi^{2}$ test and Fisher's exact test were performed. In case of a significant finding, we planned to apply Benjamini and Hochberg correction for multiple testing in order to control for the false discovery rate; however, no significant relationship was found. All analyses were performed in IBM SPSS Statistics for Windows, version 25 (IBM SPSS Statistics for Windows, Armonk, NY, USA).

\section{Results}

From 50 patients enrolled in the study, samples from $43(86 \%)$ patients could be analyzed by FTIRI, while in 4 patients, samples could not be harvested during surgery, and in 3 cases, samples were not evaluable. The mean age of patients was 72.5 (SD 7.8) years. An overview about indications for surgery is provided in Table 1.

Triple helical domain, indicative for collagen in FTIRI, showed the highest prevalence in the samples (ranging 
Fig. 1. FTIRI analysis of an ERM sample of a patient with an idiopathic ERM: native tissue sample (upper panel), organic matrix distribution based on FTIRI (middle panel), and second derivative analysis with peaks at wavenumber 1661, indicative for triple helix domain (lower panel). FTIRI, Fourier-transform infrared imaging; ERM, epiretinal membrane.

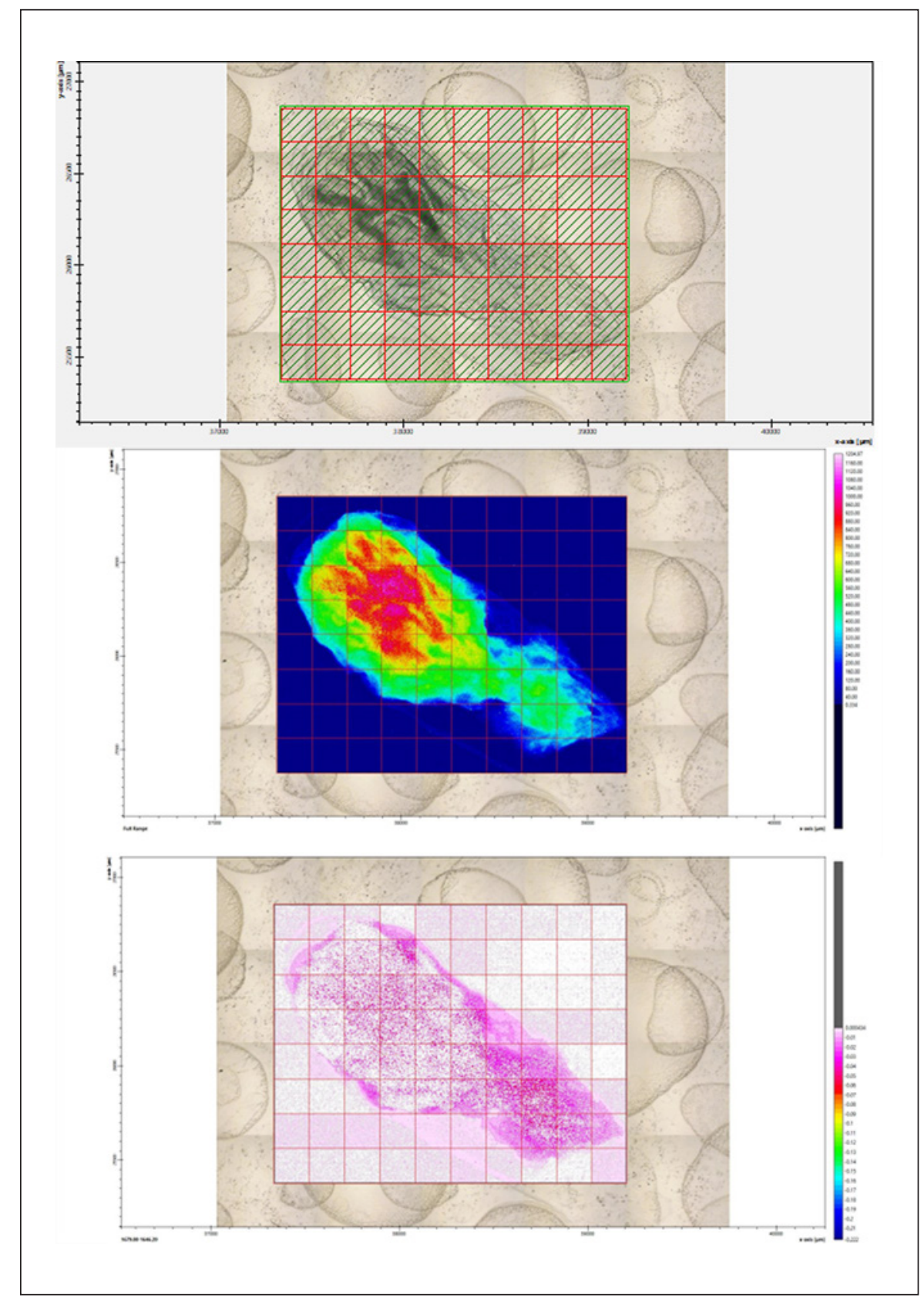

from 75 to 100\%), whereas other protein conformations did not reach such high levels of presence in the samples, with the exception of beta-turn domain in samples from atrophic lamellar macular holes (Table 1; Fig. 1).

Statistical analysis of results showed no significant associations between conformations found in FTIRI and indications for surgery (idiopathic ERM, diabetic ERM, full-thickness macular hole, atrophic lamellar macular hole, ERM foveoschisis, and pseudomacular hole), co- morbidities (such as arterial hypertension, hypercholesterinemia, and diabetes mellitus), retinal properties (such as adherence of membranes [fully or partly adherent] and intraretinal cystoid changes, both diagnosed with optical coherence tomography), and intrasurgical findings (such as good or poor staining of membranes with chromovitrectomy dye, and whether only ERM was peeled, ERM and ILM were peeled separately, or ERM and ILM were peeled en bloc) (Table 2). 
Table 2. Associations between diagnosis and general factors to secondary structures of proteins

\begin{tabular}{llllll}
\hline & $\begin{array}{l}\text { Beta-sheet } \\
\text { domain, } p\end{array}$ & $\begin{array}{l}\text { Random coil } \\
\text { domain, } p\end{array}$ & $\begin{array}{l}\text { Alpha-helical } \\
\text { domain, } p\end{array}$ & $\begin{array}{l}\text { Triple helical } \\
\text { domain, } p\end{array}$ & $\begin{array}{l}\text { Beta-turn } \\
\text { domain, } p\end{array}$ \\
\hline iERM & $0.96^{1}$ & $0.68^{2}$ & $1.0^{2}$ & $1.0^{2}$ & $0.53^{1}$ \\
dERM & $0.31^{2}$ & $1.0^{2}$ & $1.0^{2}$ & $0.56^{2}$ & $0.5^{2}$ \\
FTMH & $0.42^{2}$ & $1.0^{2}$ & $1.0^{2}$ & $0.5^{2}$ & $0.4^{2}$ \\
Atrophic LMH & $0.6^{2}$ & $0.35^{2}$ & $0.49^{2}$ & $1.0^{2}$ & $0.29^{2}$ \\
ERM foveoschisis & $1.0^{2}$ & $1.0^{2}$ & $1.0^{2}$ & $0.31^{2}$ & $0.62^{2}$ \\
Pseudomacular hole & $0.49^{2}$ & $1.0^{2}$ & $1.0^{2}$ & $1.0^{2}$ & $0.13^{2}$ \\
Arterial hypertension & $0.8^{1}$ & $0.38^{2}$ & $0.47^{2}$ & $1.0^{2}$ & $0.39^{1}$ \\
Hypercholesterinemia & $0.34^{2}$ & $1.0^{2}$ & $1.0^{2}$ & $1.0^{2}$ & $0.28^{2}$ \\
Diabetes mellitus & $0.18^{2}$ & $1.0^{2}$ & $1.0^{2}$ & $1.0^{2}$ & $0.49^{2}$ \\
Adherence of membranes & $0.78^{1}$ & $0.67^{2}$ & $0.46^{2}$ & $1.0^{2}$ & $0.94^{1}$ \\
Intraretinal cystoid changes & $0.86^{1}$ & $1.0^{2}$ & $0.68^{2}$ & $1.0^{2}$ & $1.0^{2}$ \\
Staining properties & $0.88^{1}$ & $0.6^{2}$ & $1.0^{2}$ & $0.56^{2}$ & $0.72^{2}$ \\
ERM, ILM, ERM + ILM en bloc & $0.51^{2}$ & $1.0^{2}$ & $0.67^{2}$ & $0.8^{2}$ & $0.51^{2}$ \\
\hline
\end{tabular}

ERM, epiretinal membrane; iERM, idiopathic epiretinal membrane; dERM, diabetic epiretinal membrane; FTMH, full thickness macular hole; LMH, lamellar macular hole; ILM, internal limiting membrane. ${ }^{1} \chi^{2}$ test. ${ }^{2}$ Fisher's exact test.

\section{Discussion}

In this prospective pilot study, we observed that all specific protein conformations, indicated by FTIRI, could be found in ERM and ILM with triple helical domain showing the highest prevalence. Nevertheless, the distribution of specific ones was heterogeneous, with no typical distribution patterns among different types of ERMs (idiopathic ERM, diabetic ERM, and different types of lamellar macular holes) and ILM. The results of our study, therefore, indicate a heterogeneous composition of proteins in these membranes, even in case of the same indications for surgery. This finding is in accordance with the fact that results concerning the presence of types of collagen in epiretinal tissue examined with immunohistochemical analysis were reported with differences between authors [3-6].

In general, collagen types I, II, III, IV, and VI were found in ERMs [3-6]. Kritzenberger et al. [3] found a high amount of collagen type VI and a relative absence of collagen types I and II in cellophane maculopathy compared to preretinal fibrosis. Bu et al. $[4,5]$ found collagen types I, II, IV, and VI in ERM samples and collagen types I, III, and V in membrane samples from macular hole surgery, whereas Compera et al. [6] found collagen types I and III in samples from lamellar macular holes. Apart from collagen, also laminin, fibronectin, and $\alpha$-SMA were found in ERMs [3, 4, 6]. Collagen types I, II, III, and V are fibril-forming collagens, with characteristic patterns of bands in electron microscopy, whereas collagen type IV is a sheet-forming collagen, typical for all basal laminas, and collagen type VI is a fibril-associated collagen, associated with collagen type I [7].

All of the abovementioned types of collagen show a triple helical configuration in the central part (for collagen types IV and VI, the central triple helices are interrupted, enabling more lateral flexibility than the fibrilforming types of collagen I, II, III, and V) with telopeptides at both ends (the $\mathrm{C}$ and $\mathrm{N}$ terminals). Triple helical domain in FTIRI is, therefore, indicative for the presence of collagen molecules. We have no explanation why the triple helical domain could not be shown in all samples. Nevertheless, ERM and ILM also consist of other proteins with possible influence on FTIRI outcomes.

As the FTIRI results resemble information of spatial characteristics of proteins and their neighborhoods in the examined tissue, other proteins in ERM and ILM either from telopeptides or the extracellular matrix have the potential to influence the results. Up to now the presence of specific conformations of proteins in epiretinal tissues, such as the beta-sheet domain, random coil domain, alpha-helical domain, and beta-turn domain, has not been examined enough to give conclusive answers with respect to that question. Due to the fact that this study was planned as a pilot study, investigating FTIRI for epiretinal tissues in general, future research will be needed to improve knowledge on protein origins and their impact on FTIRI outcomes. Samples were examined in a native state to avoid possible denaturation of proteins through fixa- 
tion with aldehydes, as this procedure has potential for creation of artifacts in FTIRI results.

We did not find any significant associations of specific conformations, identified by FTIRI, to the indications for surgery, comorbidities of patients, retinal properties, and intrasurgical findings most likely due to low patient numbers, as this study was designed as an exploratory one (Table 2). FTIRI results, however, provide a completely new mode of examination of excised tissues in ophthalmology, and further research is needed to better understand the clinical impact of the spectroscopic findings.

A limitation of the study is the fact that the study was planned and performed as a pilot study with the former classification of lamellar macular holes, and, therefore, numbers of samples from patients with atrophic lamellar macular holes, ERM foveoschisis, and pseudomacular holes are low. Nevertheless, $100 \%$ presence of beta-turn domain and triple helical domain in atrophic lamellar holes, and the fact that in pseudomacular holes only triple helical domain could be found are novel results with the need to be validated with a higher number of samples. In conclusion, FTIRI is a new method of analysis of ERMs and ILM and their composition of proteins, with spatial information including specific conformation of molecules.

\section{Acknowledgements}

The authors would like to acknowledge the Adele Rabensteiner Foundation of the Austrian Society of Ophthalmology for financially supporting the study.

\section{Statement of Ethics}

All research and measurements followed the tenets of the Declaration of Helsinki and were approved by the local Ethics Committee of the city of Vienna (Approval number: EK-17-089-0517). Written informed consent was obtained from all patients in the study.

\section{Conflict of Interest Statement}

O. Findl is a scientific advisor for Alcon, Croma, Carl Zeiss Meditec AG, Johnson \& Johnson, and Merck, but has no personal interest in the products mentioned. All authors declare that there are no conflicts of interest.

\section{Funding Sources}

The study was supported by a grant from the Adele Rabensteiner Foundation, Austria. The Adele Rabensteiner Foundation did not influence the outcomes of the study or manuscript preparation in any way.

\section{Author Contributions}

C. Leisser, ${ }^{1-4}$ E. Paschalis, ${ }^{1,2,5}$ S. Rokidi, ${ }^{2,5}$ M. Behanova, ${ }^{3,5}$ M. Ruiss, ${ }^{2,5}$ W. Burgmüller, ${ }^{2,5}$ and O. Findl ${ }^{1,5}$ ( ${ }^{1}$ conception of the study, ${ }^{2}$ data acquisition, ${ }^{3}$ statistical analysis, ${ }^{4}$ preparation of the manuscript, and ${ }^{5}$ critical review of the manuscript).

\section{References}

1 Torii $\mathrm{H}$, Tasumi M. Theoretical analyses of the amide I infrared bands of globular proteins. In: Mantsch HH, Chapman D, editors. Infrared spectroscopy of biomolecules. New York, NY: Wiley-Liss; 1996. p. 1-18.

2 Byler DM, Susi H. Examination of the secondary structure of proteins by deconvolved FTIR spectra. Biopolymers. 1986 Mar;25(3): 469-87.

3 Kritzenberger $M$, Junglas B, Framme C, Helbig H, Gabel VP, Fuchshofer R, et al. Different collagen types define two types of idio- pathic epiretinal membranes. Histopathology. 2011 May;58(6):953-65.

4 Bu SC, Kuijer R, van der Worp RJ, Postma G, Renardel de Lavalette VW, Li XR, et al. Immunohistochemical evaluation of idiopathic epiretinal membranes and in vitro studies on the effect of TGF- $\beta$ on müller cells. Invest Ophthalmol Vis Sci. 2015 Oct;56(11):650614.

5 Bu S-C, Kuijer R, van der Worp RJ, Huiskamp EA, Renardel de Lavalette VW, Li X-R, et al. Glial cells and collagens in epiretinal mem- branes associated with idiopathic macular holes. Retina. 2014 May;34(5):897-906.

6 Compera D, Entchev E, Haritoglou C, Scheler R, Mayer WJ, Wolf A, et al. Lamellar holeassociated epiretinal proliferation in comparison to epiretinal membranes of macular pseudoholes. Am J Ophthalmol. 2015 Aug; 160(2):373-84e1

7 Kuhn K. In: Mayne D, Burgeson R, editors. Structure and Function of Collagen Types. Academic Press; 1987. van der Rest M, Garrone R. FASEB J. 1991;5:22814. 